\title{
MAPEAMENTO DOS CASOS DE DENGUE NO PONTAL DO PARANAPANEMA NO PERÍODO DE 2007 A 2012
}

\author{
MAPPING OF DENGUE CASES IN PONTAL OF PARANAPANEMA FROM \\ 2007 TO 2012
}

Letícia Aparecida Costa ${ }^{1}$; Mayara Maezano Faita Pinheiro ${ }^{2}$; Vitor Guilherme Saccomani Servilha'; Mario Boscoli Neto ${ }^{2}$; Jacqueline Roberta Tamashiro ${ }^{1}$; Ana Paula Marques Ramos ${ }^{1}$

Universidade do Oeste Paulista - UNOESTE, ${ }^{1}$ Mestrado em Meio Ambiente e Desenvolvimento Regional; ${ }^{2}$ Aluna(o) especial do mestrado em Meio Ambiente e Desenvolvimento Regional, Presidente Prudente - SP.

E-mail' lecosta.amb@gmail.com

RESUMO - Doenças transmitidas por mosquitos estão cada vez mais frequentes e diversificadas em todo mundo. Isto é provável que esteja apenas no estágio inicial de ondas epidêmicas. O objetivo deste trabalho é mapear os casos de dengue na região do Pontal do Paranapanema no período de 2007 a 2012. Devido à falta de informações sobre a dengue no Pontal, a principal contribuição deste trabalho é auxiliar na visualização de quais municípios correspondem às áreas mais críticas dessa doença e se há correlação da mesma com as questões de urbanização e de saneamento do município. A distribuição espacial dos casos de dengue foram representadas em mapas temáticos do tipo coroplético. Conclui-se que a urbanização possui uma relação direta com o aumento dos casos de dengue, visto que municípios com maiores índices de urbanização são também com maiores taxas de dengue. Outros resultados serão discutidos.

Palavras-chave: Dengue; Mapas temáticos; Pontal do Paranapanema.

ABSTRACT - Diseases transmitted by mosquitoes are becoming more frequent and diverse in the world. It is possible to be only in the early stages of epidemic waves. This work aims to mapping cases of dengue in the Pontal do Paranapanema region from 2007 to 2012. Due to a lack of information about dengue in Pontal the main contribution of this work consists of visualize which are municipalities with the highest rates of dengue cases and if there is some correspondence with the urbanization and sanitation factors. The spatial distribution of dengue cases was mapped using thematic maps such as choropleth kind. We

Recebido em: 19/08/2016 Revisado em: 23/08/2016 Aprovado em: 25/08/2016 concluded that urbanization and dengue factors are related because the municipalities with the highest urbanization index are also those with high rates of dengue cases. Others results are discussed.

Keywords: Dengue; Thematic Maps; Pontal do Paranapanema. 


\section{INTRODUÇÃO}

A dengue é considerada um grave problema de saúde pública nas regiões tropicais e subtropicais do planeta, devido à complexidade de fatores que a envolve, como profilaxia, controle e tratamento, bem como por seu significativo índice de mortalidade e morbidade (SUAREZ et al, 2009; RESENDES et al, 2010; WHO, 2016; BHATT et al, 2013). Mundialmente a dengue apresenta crescimento na faixa de incidências nas últimas décadas, especialmente, nos países de clima tropical. De acordo com a Organização Mundial Da Saúde (OMS), 2,5 bilhões de pessoas correm o risco de contrair a doença, ou seja, mais de 40\% da população mundial (WHO, 2016). Esta é uma doença endêmica presente em 115 países - localizados principalmente no sudeste asiático e na América Latina; estimase que no mundo todo existam cerca de 50 a 100 milhões de infectados anualmente e cerca de 24 mil casos são fatais (WHO, 2016).

É cada vez mais frequente e mais diversificada em todo mundo doenças transmitidas por mosquitos e é provável que essas estejam apenas no estágio inicial de ondas epidêmicas que vai continuar por várias décadas (MISSIER et al 2016). Devido à propagação rápida de doenças transmitidas pelos mosquitos Aedes aegypti, que carregam não apenas o vírus da dengue, mas também vírus Chikungunya e Zika Vírus é necessário cuidado rigoroso. (MISSIER et al 2016).

Segundo Dias (2010), a dengue é uma arbovirose, sendo transmitida principalmente pela picada do mosquito Aedes aegypti. Esta doença pode ser assintomática ou apresentar amplo sintoma clínico, variando de doença febril autolimitada até sintomas graves, que podem evoluir com choque circulatório e óbito (DIAS, 2010).

Diversos fatores de risco estão relacionados com presença da doença (dengue) e do vetor (Aedes aegypti). Tauil (2001) destaca como fatores fundamentais para definir o padrão de transmissão: crescimento populacional, migrações, viagens aéreas, urbanização inadequada, mau funcionamento dos sistemas de saúde e densidade populacional. De acordo com De Simone, et al. (2004), a análise da dispersão da infecção através de sistemas interligados de populações, tais como centros urbanos, é de grande importância e atrai considerável interesse, em especial, para o planejamento de resposta às doenças emergentes pandêmicas. 0 padrão temporal epidemiológico da dengue é caracterizado por epidemias periódicas com ciclos interepidêmicos. Isto mostra variações sazonais no tamanho da população do vetor e com prevalências de determinados sorotipos que variam na dominância ao longo 
do tempo e do espaço (DEGALLIER et al, 2009 apud HORTA et al, 2013).

De acordo com Horta et al (2013), a análise da paisagem, utilizando técnicas de geoprocessamento, pode fornecer ferramentas para um melhor planejamento urbano, permitindo uma melhor qualidade de vida e preservação de áreas naturais. Surtos de dengue em grandes cidades têm, entre outros fatores a expansão urbana, o número elevado de locais de reprodução do mosquito, as altas temperaturas e o grande número de pessoas suscetíveis. Neste sentido, o mapeamento de doenças tem sido um instrumento importante no campo da saúde pública. Desde os anos 1990, as técnicas de análise foram melhoradas para gerar mapas de identificação de áreas de risco, resultando em atenção diferenciada a ser dada pelos serviços de saúde (SIQUEIRA et al, 2008). Tais mapas têm sido amplamente utilizados para compreender a dinâmica de doenças infecciosas (SAZONOV et al, 2008; COELHO et al, 2008).

O objetivo desse trabalho é mapear a taxa média dos casos de dengue, no período de 2007 a 2012, na região do Pontal do Paranapanema, Estado de São Paulo. O objeto de estudo refere-se aos 32 municípios que constituem o Pontal. As questões que este trabalho responde são: ‘Há uma distribuição homogênea do número de casos de dengue nos municípios da região do
Pontal do Paranapanema' e 'Fatores como urbanização e densidade populacional podem ser associados à ocorrência dos casos de dengue?'. Devido à falta de informações sobre a ocorrência de casos de dengue no objeto de estudo, a principal contribuição desse trabalho é auxiliar no estudo do fenômeno geográfico.

\section{1. ÁREA DE ESTUDO}

A Região do Pontal do Paranapanema, localizada no extremo oeste do Estado de São Paulo, é composta por 32 municípios que abrangem uma área total de $18.396,423 \mathrm{~km}^{2}$. O município que possui a maior área de unidade territorial é Rancharia, com $1.587,498 \mathrm{~km}^{2}$ de extensão total, enquanto a menor área apresentada é a do Município de Santo Expedito, com 94,465 $\mathrm{km}^{2}$ (IBGE, 2010).

A Figura 1 apresenta a localização dos 32 Municípios inseridos na Região do Pontal do Paranapanema, que é considerada privilegiada do ponto de vista logístico, pois está inserida entre os Estados do Paraná e Mato Grosso do Sul, com vias de acesso que propiciam a integração entre estes estados e dão acesso a pontos estratégicos para regiões produtoras e grandes centros. Já no aspecto econômico a "econômica regional é caracterizada pela integração entre as atividades primárias e secundárias" e a agroindústria é o principal segmento baseado 
no agronegócio, seguido pelo processamento de carne e produção sucroalcooleira (açúcar e álcool) (BRASIL, 2013). 


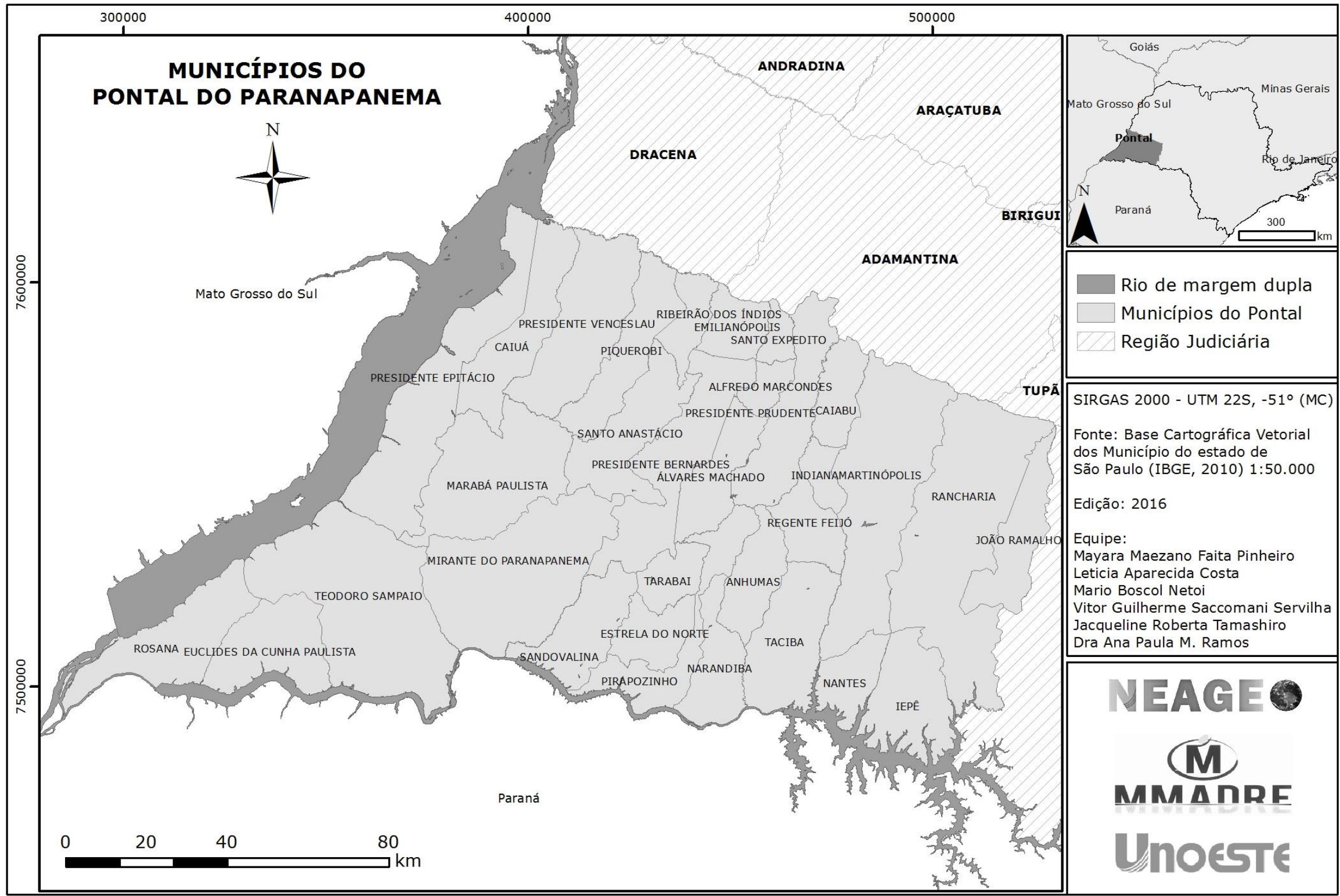

Figura 1. Municípios do Pontal do Paranapanema Fonte: Autores, 2016. 


\section{METODOLOGIA}

O método foi dividido em três etapas: levantamento dos dados de dengue na base de dados do DATASUS (Departamento de Informática do Sistema Único de Saúde) (BRASIL, 2016), de responsabilidade do Ministério da Saúde, e levantamento de dados sobre a população urbana e rural, e de saneamento, no Censo de 2010 do IBGE (Instituto Brasileiro de Geografia e Estatística); organização dos dados utilizando o Microsoft Office Excel; e elaboração dos mapas temáticos pelo software de informação geográfica, QGIS

2.14. Elaboraram-se mapas temáticos tanto para representar a taxa média de dengue entre 2007 e 20012, quanto às condições de saneamento e a distribuição da população rural e população urbana nos 32 municípios em estudo.

\subsection{LEVANTAMENTO E ORGANIZAÇÃO DOS DADOS}

Os dados dos casos de dengue no Pontal do Paranapanema foram obtidos no DATASUS e através do link "TABNET", conforme apresentado na Figura 2.

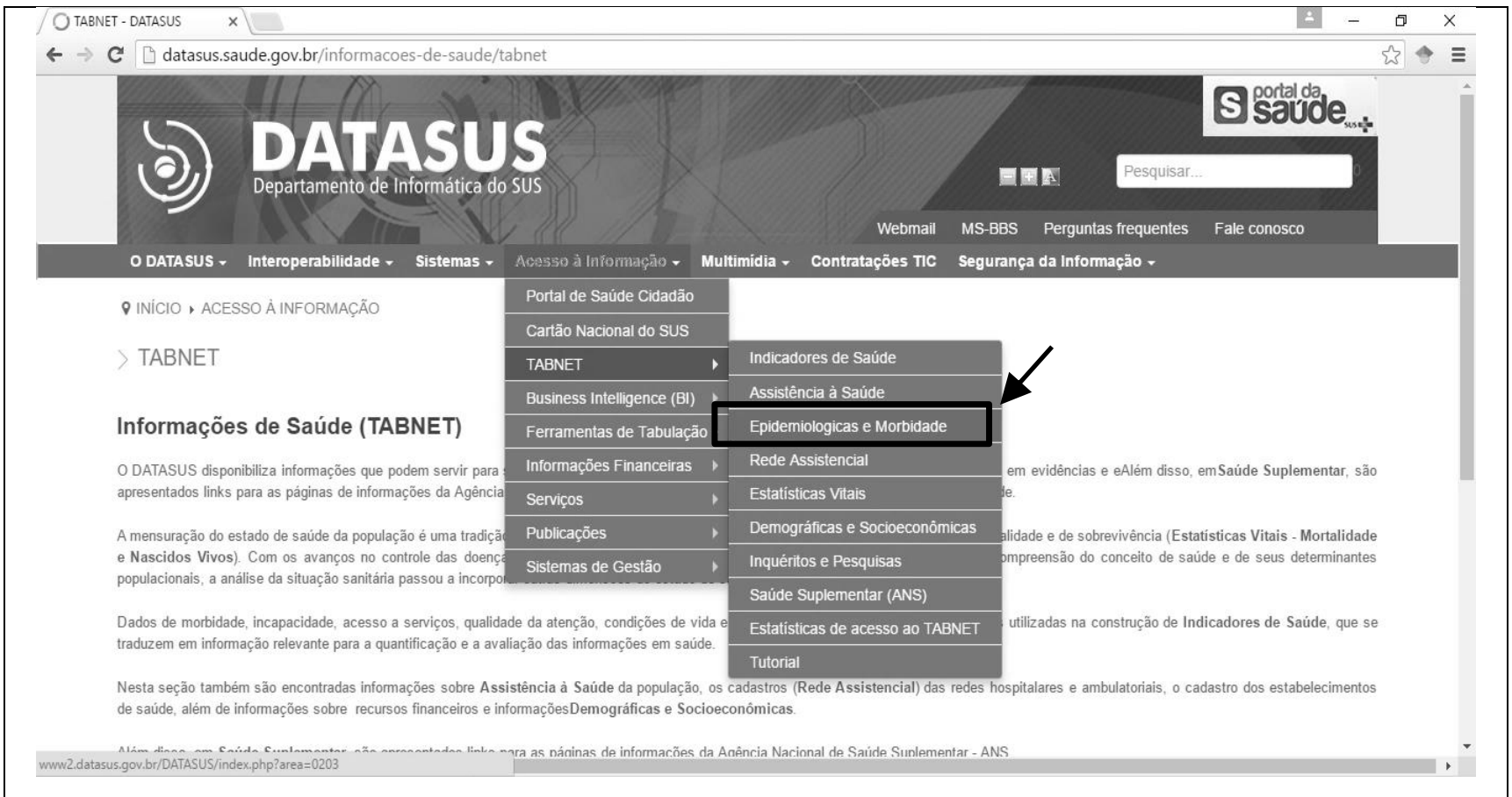

Figura 2. Tela do DATASUS TABNET - Acesso à informação

Fonte: DATASUS (2016).

Para filtrar os dados para a região de interesse o sistema disponibiliza a opção de pesquisa com classificação de tipos de doenças e agravos, bem como, disponibiliza a escolha da abrangência geográfica para o
Brasil, conforme ilustra a Figura 3. As opções escolhidas para captação dos dados desejados e nesse trabalho estão apresentadas na Figura 4. 
Foram coletados dados sobre a quantidade de população urbana e rural no Pontal do Paranapanema e dados sobre a qualidade do saneamento básico em cada município segundo o Censo de 2010 que foram obtidos no site do IBGE. Os dados de população são disponibilizados em porcentagem, os dados de saneamento inadequado, sendo aquelas residências onde não há abastecimento de água por rede geral, esgotamento sanitário por rede geral ou fossa séptica e lixo coletado diretamente ou indiretamente adequados e abrangentes, segundo o IBGE (2010). Os casos de dengue por municípios foram estimados em termos de taxa a cada cem mil habitantes.

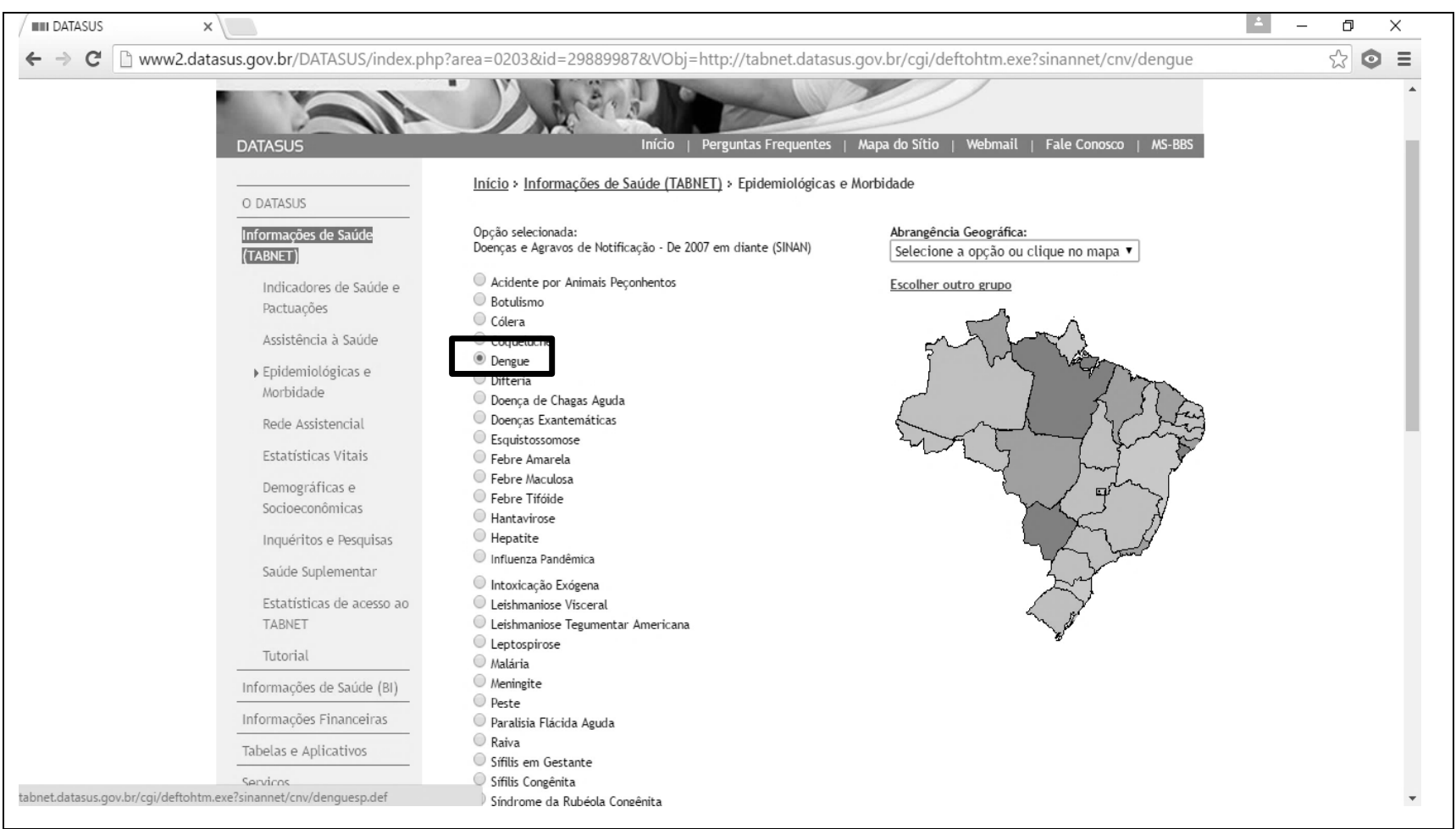

Figura 3. Seleção de opção e abrangência geográfica Fonte: DATASUS (2016). 


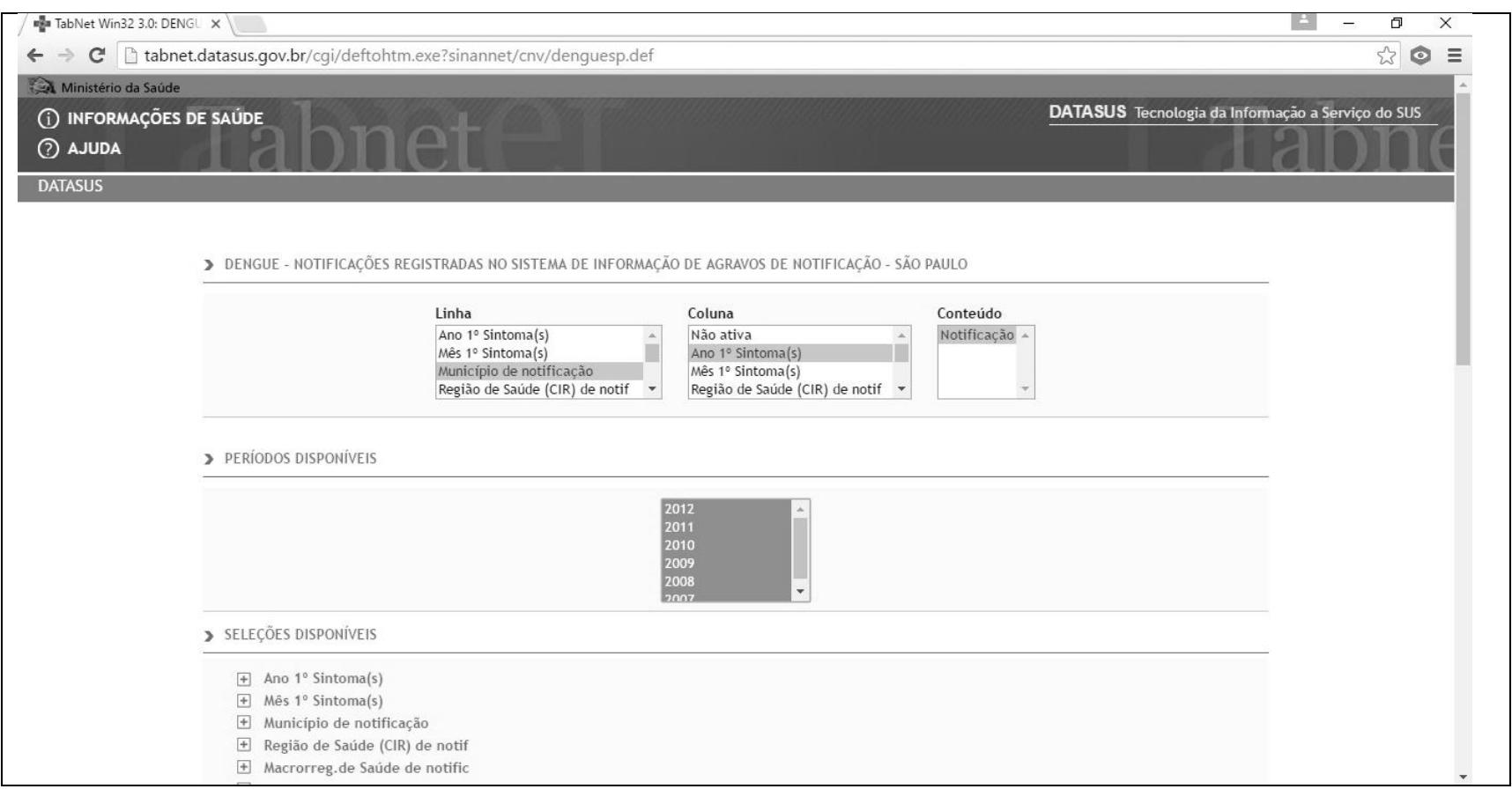

Figura 4. Seleção dos dados para o período de 2007 a 2012

Fonte: DATASUS (2016).

A estimativa da taxa por 100.000, de 2007 a 2012, é expressa na equação (I):

$$
\frac{\text { Número de casos }}{\text { População Município }} \times 100.000
$$

Os casos de dengue, saneamento e população foram organizados em planilha Excel, para posterior elaboração dos mapas. Os dados organizados encontram-se na Tabela 1. 
Tabela 1. Organização dos Dados

\begin{tabular}{|c|c|c|c|c|c|c|c|}
\hline A & B & C & D & E & $\mathbf{F}$ & G & $\mathbf{H}$ \\
\hline 3500808 & Alfredo Marcondes & 3.891 & 6 & 154,2 & $10 \%$ & $84 \%$ & $16 \%$ \\
\hline 3501301 & Álvares Machado & 23.513 & 108 & 459,3 & $6 \%$ & $90 \%$ & $10 \%$ \\
\hline 3502408 & Anhumas & 3.738 & 1 & 26,8 & $12 \%$ & $82 \%$ & $18 \%$ \\
\hline 3508900 & Caiabú & 4.072 & 3 & 73,7 & $11 \%$ & $81 \%$ & $19 \%$ \\
\hline 3509106 & Caiuá & 5.039 & 10 & 198,5 & $30 \%$ & $38 \%$ & $62 \%$ \\
\hline 3515129 & Emilianópolis & 3.020 & 3 & 99,3 & $11 \%$ & $83 \%$ & $17 \%$ \\
\hline 3515301 & Estrela do Norte & 2.658 & 1 & 37,6 & $13 \%$ & $79 \%$ & $21 \%$ \\
\hline 3515350 & Euclides da Cunha & 9.585 & 44 & 459,1 & $28 \%$ & $64 \%$ & $36 \%$ \\
\hline 3519907 & lepê & 7.628 & 0 & 0 & $7 \%$ & $89 \%$ & $11 \%$ \\
\hline 3520608 & Indiana & 4.825 & 16 & 331,6 & $7 \%$ & $86 \%$ & $14 \%$ \\
\hline 3525607 & João Ramalho & 4.150 & 5 & 120,5 & $11 \%$ & $85 \%$ & $15 \%$ \\
\hline 3528700 & Marabá Paulista & 4.812 & 9 & 187 & $32 \%$ & $45 \%$ & $55 \%$ \\
\hline 3529203 & Martinópolis & 24.219 & 37 & 152,8 & $4 \%$ & $84 \%$ & $16 \%$ \\
\hline 3530201 & Mirante do Paranapanema & 17.059 & 90 & 527,6 & $30 \%$ & $59 \%$ & $41 \%$ \\
\hline 3532157 & Nantes & 2.707 & 0 & 0 & $2 \%$ & $90 \%$ & $10 \%$ \\
\hline 3532207 & Narandiba & 4.288 & 26 & 606,3 & $21 \%$ & $72 \%$ & $28 \%$ \\
\hline 3538303 & Piquerobi & 3.537 & 21 & 593,7 & $21 \%$ & $75 \%$ & $25 \%$ \\
\hline 3539202 & Pirapozinho & 24.694 & 23 & 93,1 & $2 \%$ & $95 \%$ & $5 \%$ \\
\hline 3541208 & Presidente Bernardes & 13.570 & 54 & 397,9 & $15 \%$ & $77 \%$ & $23 \%$ \\
\hline 3541307 & Presidente Epitácio & 41.318 & 747 & $1.807,90$ & $6 \%$ & $93 \%$ & $7 \%$ \\
\hline 3541406 & Presidente Prudente & 207.610 & 1228 & 591,5 & $1 \%$ & $98 \%$ & $2 \%$ \\
\hline 3541505 & Presidente Venceslau & 37.910 & 400 & $1.055,10$ & $3 \%$ & $96 \%$ & $4 \%$ \\
\hline 3542206 & Rancharia & 28.804 & 152 & 527,7 & $5 \%$ & $90 \%$ & $10 \%$ \\
\hline 3542404 & Regente Feijó & 18.494 & 55 & 297,4 & $4 \%$ & $92 \%$ & $8 \%$ \\
\hline 3543238 & Ribeirão dos Índios & 2.187 & 3 & 137,2 & $12 \%$ & $85 \%$ & $15 \%$ \\
\hline 3544251 & Rosana & 19.691 & 105 & 533,2 & $13 \%$ & $81 \%$ & $19 \%$ \\
\hline 3545506 & Sandovalina & 3.699 & 4 & 108,1 & $28 \%$ & $70 \%$ & $30 \%$ \\
\hline 3547700 & Santo Anastácio & 20.475 & 130 & 634,9 & $5 \%$ & $93 \%$ & $7 \%$ \\
\hline 3548302 & Santo Expedito & 2.803 & 5 & 178,4 & $4 \%$ & $88 \%$ & $12 \%$ \\
\hline 3552908 & Taciba & 5.714 & 9 & 157,5 & $11 \%$ & $85 \%$ & $15 \%$ \\
\hline 3553906 & Tarabai & 6.607 & 10 & 151,4 & $4 \%$ & $92 \%$ & $8 \%$ \\
\hline 3554300 & Teodoro Sampaio & 21.386 & 369 & $1.725,40$ & $17 \%$ & $81 \%$ & $19 \%$ \\
\hline
\end{tabular}

$\mathrm{A}^{1}$ - Código do Município segundo o IBGE.

$\mathrm{B}^{2}$ - Nome do Município.

$\mathrm{C}^{3}$ - População segundo o Censo do IBGE de 2010.

$D^{4}$ - Total de Casos de dengue no período de 2007 a 2012.

$\mathrm{E}^{5}$ - Taxa média de dengue a cada 100 mil habitantes no período de 2007 a 2012

$\mathrm{F}^{6}$ - Porcentagem de Residências com Saneamento Inadequado segundo o Censo do IBGE de 2010.

$\mathrm{G}^{7}$ - Porcentagem de população Urbana segundo o Censo do IBGE de 2010.

$\mathrm{H}^{8}$ - Porcentagem de população Rural segundo o Censo do IBGE de 2010.

Fonte: IBGE (2010); DATASUS (2016).

Organização: Autores (2016).

\subsection{ELABORAÇÃO DOS MAPAS}

Elaborou-se um total de três mapas temáticos, quais sejam:

- Mapa 1 - Distribuição da taxa média de dengue entre 2007 e 2012 no Pontal do Paranapanema;
- Mapa 2 - Porcentagem de residências com Saneamento Inadequado no Pontal do Paranapanema; e 
- Mapa 3 - Distribuição da taxa média de dengue entre 2007 e 2012 com a distribuição da população urbana e rural no Pontal do Paranapanema.

O método de representação adotado foi o coroplético, isto porque se tratam de dados quantitativos relativos, sendo, portanto, o tipo de representação temática mais apropriada conforme Dent et al (2009). A divisão das classes foi realizada através do método otimização de Jenks. Este método é caracterizado por formar classes homogêneas internamente entre si e assegurar a heterogeneidade entre as classes (DENT et al, 2009).

Cabe ressaltar que o mapa de distribuição da população foi elaborado tendo como base o mapa de distribuição da taxa média de dengue entre 2007 e 2012. Neste caso, o porcentual de população urbana e população rural foi representada por meio de gráficos em formato de pizza para cada município.

\section{RESULTADOS E DISCUSSÃO}

A Figura 5 ilustra o mapa de distribuição da taxa média de dengue entre 2007 e 2012 nos 32 municípios do Pontal do Paranapanema. Nota-se (Figura 5) que não existe uma homogeneidade da taxa de dengue distribuição da dengue nos municípios em estudo. Os maiores valores ocorrem nos municípios de Teodoro Sampaio e Presidente Epitácio com taxas variando de 1055, 2 a 1807,9 por 100 mil habitantes. Em seguida, encontram-se nove municípios (Mirante do Paranapanema, Narandiba, Presidente Bernardes, Presidente Prudente, Presidente Venceslau, Piquerobi, Santo Anastácio, Rancharia, Rosana). Dentre esses nove municípios que apresentaram a segunda maior taxa e que merecem destaque, estão Presidente Prudente por ser o mais populoso do Pontal, e o município de Rancharia por ser o de maior extensão.

Apenas dois municípios não tiveram registros de casos de dengue quais sejam Nantes e lepê. Uma razão pode ser o atraso no registro e compilação dos dados para serem divulgados. 


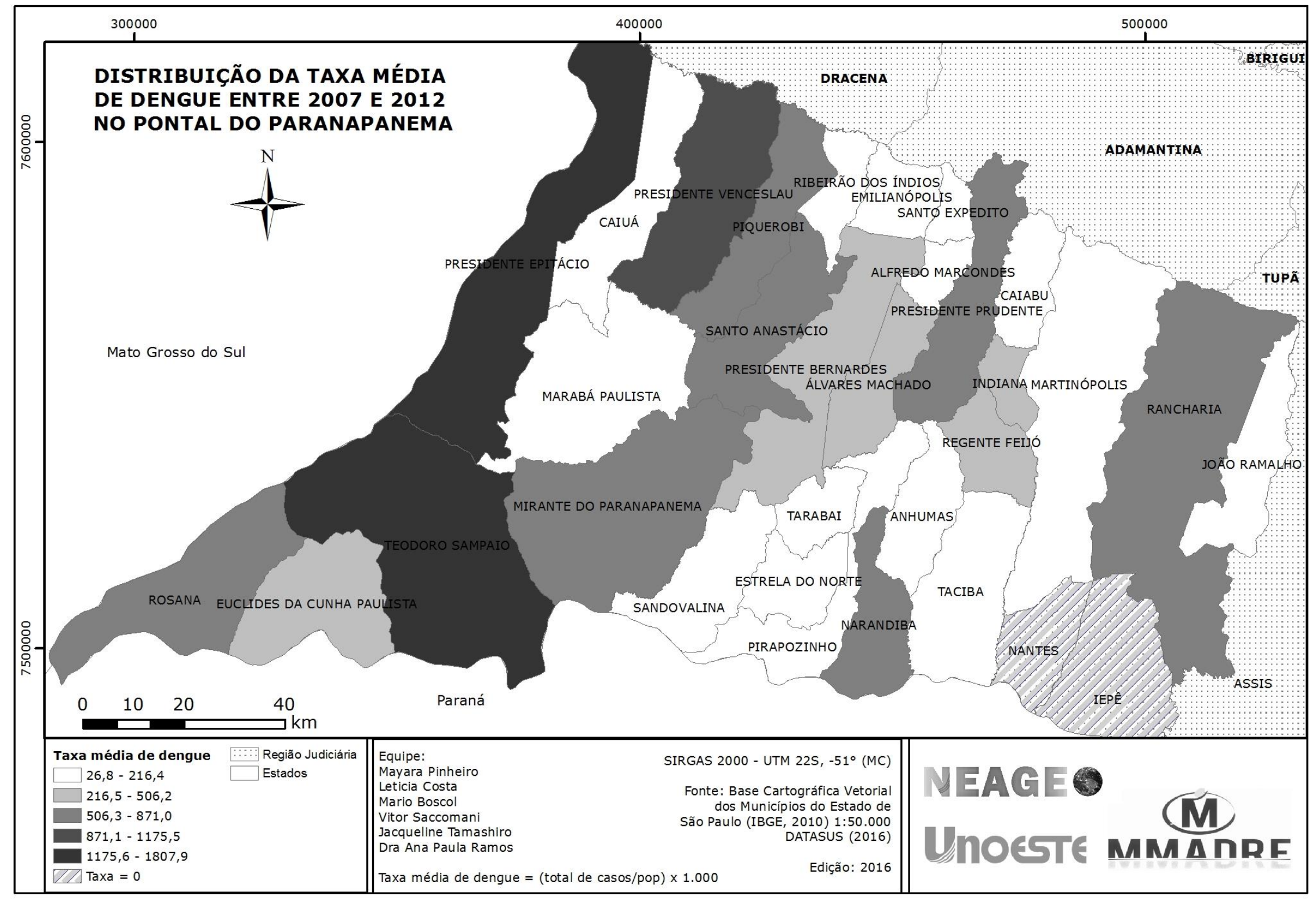

Figura 5. Distribuição da taxa média de dengue entre 2007 e 2012 no Pontal do Paranapanema Fonte: Autores (2016). 
A Figura 6 apresenta o mapa de saneamento, ou seja, o mapa de \% de residências nos municípios do Pontal com saneamento básico considerado inadequado de acordo com o IBGE (2010). Conforme Figura 6 as maiores quantidades foram encontrados nos municípios de Euclides da Cunha Paulista, Caiuá, Marabá Paulista, Mirante do Paranapanema, Sandovalina, Narandiba, e Piquerobi.

Ao analisar o mapa da Figura 6 com o mapa de distribuição da taxa média de dengue (Figura 7), pode-se afirmar que 0 saneamento básico não se mostra como um fator predominante para se contrair a dengue. Em alguns municípios pode-se inferir alguma associação, como em Mirante que possui elevados casos de dengue e maior porcentagem de residências com saneamento inadequado, porém em Presidente Prudente, por exemplo, tem-se uma elevada taxa de dengue (Figura 6) e com menor porcentagem de residências de saneamento inadequado (Figura 7).

O Aedes aegypti está completamente adaptado ao habitat urbano, onde encontra adjacente aos domicílios, as condições necessárias para o seu desenvolvimento (SILVA; MARIANO; SCOPEL, 2008). Para tentar responder ao questionamento sobre a associação da doença e os fatores de urbanização, a Figura 8 apresenta o mapa de distribuição da dengue comparando a distribuição da população urbana e rural nos 32 municípios em estudo.

Observa-se a partir desse mapa (Figura 7) se os casos de dengue tem relação com a distribuição da população. É evidente que municípios com altas taxas de dengue, como Presidente Prudente, Presidente Bernardes, Presidente Venceslau e Rancharia, possuem uma grande parcela de sua população localizada em área urbana. Alguns municípios como Marabá Paulista e Caiuá apresentam uma baixa taxa de dengue e a população rural é muito próxima da urbana.

Os municípios da Região do Pontal do Paranapanema, de forma geral, apresentam cerca de $80 \%$ da população localizada na área urbana e apenas $30 \%$ situada na área rural. 0 Município que apresenta a maior porcentagem da população em área urbana é o de Presidente Prudente com 98\% da população, enquanto o que apresenta a maior parte da população inserida na área rural, com 62\% é o Município de Caiuá.

Essa associação entre dengue $\mathrm{e}$ população não pode ser definida como um padrão para todos os municípios do Pontal, mas aponta concentrações da população que pode influenciar os agravos de casos de dengue. Isso pode estar correlacionado com o principal "foco" de transmissão da doença que é a água parada. 


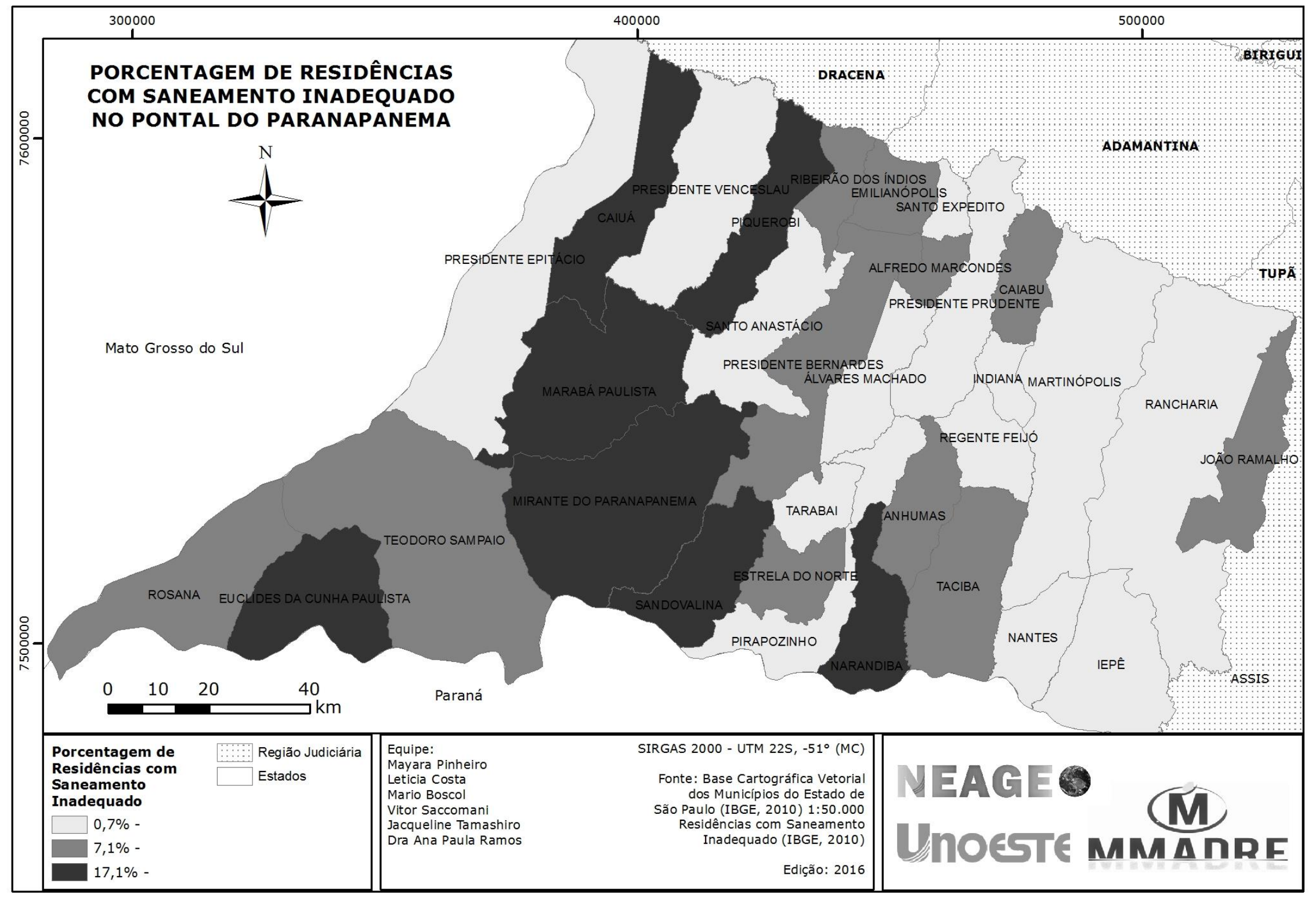

Figura 6. Porcentagem de residências com saneamento inadequado no Pontal do Paranapanema Fonte: Autores (2016). 


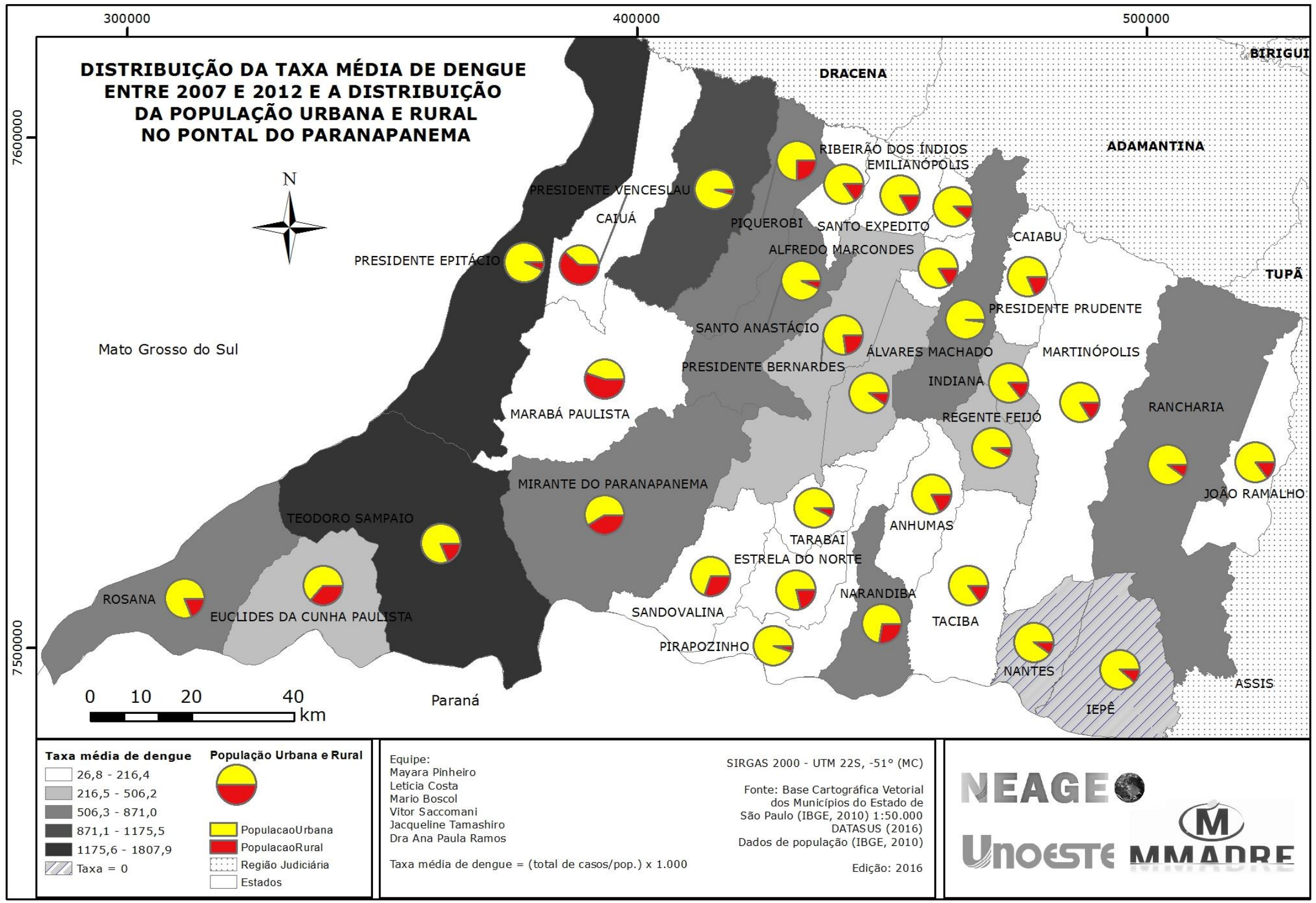

Figura 7. Distribuição da taxa média de dengue entre 2007 e 2012 e a distribuição da população urbana e rural no Pontal do Paranapanema Fonte: Autores (2016). 
Nas áreas rurais a água infiltra o solo, pois a maior parte do solo não está impermeabilizado, minimizando esses locais que são criadouros para o mosquito. Diferentemente das cidades, onde há grande abrangência de pavimentação impermeável facilitando que a água se acumule nessas regiões. Nos centros urbanos também deparamo-nos com a disposição inadequada de resíduos, como pneus, que é um veículo para a água se acumular.

O conhecimento da produtividade dos diversos tipos de criadouros em meio urbano e dos condicionantes ambientais, bem como a presença e a dinâmica do vírus da dengue são de grande relevância para se compreender a dinâmica da transmissão da doença (MEDRONHO, 2006). Para os autores Mendonça et al. (2009), o processo de urbanização e a cidade, são contingências fundamentais para o aumento e intensificação de processos epidêmicopandêmicos nas mais diferentes localidades regionais.

De acordo com Catão (2012), a relação entre doença e produção espacial é uma variável a ser considerada na análise da doença, por ser o principal vetor, um mosquito predominantemente urbano e com acentuada predileção pelo sangue humano ou antropofílico, o Aedes aegypti é adaptado ao espaço geográfico, aos objetos geográficos e aos fluxos de pessoas e materiais.

\section{CONSIDERAÇÕES FINAIS}

A partir dos mapas elaborados foi possível visualizar a quantidade (taxa média) de casos de dengue nos municípios do Pontal do Paranapanema, bem como, se há relação dessa doença com o saneamento inadequado e a quantidade de população urbana e rural. Conclui-se que a distribuição de dengue no Pontal do Paranapanema, no período de 2007 a 2012, em termos de taxas médias, não se apresenta homogênea nos 32 municípios estudados. Além disso, o fator saneamento inadequado não apresentou correlação com a ocorrência dessa doença. Todavia, na maioria dos municípios, a quantidade de população urbana esteve diretamente relacionada com as maiores taxas médias de dengue.

Ressalta-se que outros fatores devem ser analisados, a fim de que se obtenham resultados mais detalhados para o fenômeno em estudo. Um exemplo, consiste em relacionar a ocorrência de dengue com o clima, a temperatura e o índice pluviométrico do Pontal.

\section{AGRADECIMENTOS}

Agradecemos ao Mestrado em Meio Ambiente e Desenvolvimento Regional 
(MMADRE), e ao Núcleo de Estudos Ambientais e Geoprocessamento (NEAGEO).

\section{REFERÊNCIAS}

BHATT, S. et al. The global distribution and burden of dengue. Nature, v.496, n.7446, p.504-7, 25 abr. 2013. https://doi.org/10.1038/nature12060

BRASIL. Ministério Da Saúde. DATASUS. Informações de saúde: dengue, 2007 a 2012. Disponível em: <http://datasus.saude.gov.br/informacoesde-saude/tabnet>. Acesso em: 14 mai. 2016.

BRASIL. Secretaria de Planejamento e Desenvolvimento Regional do Estado de São Paulo. Caracterização socioeconômica das regiões do Estado de São Paulo- Região Administrativa de Presidente Prudente, mar. 2013.

CATÃO, R. C. Dengue no Brasil: abordagem geográfica na escala nacional. São Paulo: Cultura Acadêmica. Sindicato Nacional Dos Editores de Livros, 2012.

COELHO, F.C.; CODEÇO, C.T.; STRUCHINER, C.J. Complete treatment of uncertainties in a model for dengue R0 estimation. Cad. Saúde Pública, Rio de Janeiro, v. 24, n. 4, p. 853861, abr. 2008.

DE SIMONE, T.S. et al. Dengue virus surveillance: the co-circulation of DENV-1, DENV-2 and DENV-3 in the state of Rio de Janeiro, Brazil. Rev. Trans. R. C. Trop. Med. Hyg., Rio de Janeiro, v.98, p. 553-562, 2004.

DEGALLIER, $N$. et al. Imported and autochthonous cases in the dynamics of dengue epidemics in Brazil. Rev. Saúde Pública, São Paulo, v.43, n. 1, p. 17, fev. 2009. https://doi.org/10.1590/S003489102009000100001
DENT, B. D.; TORGUSON, J.; HODLER, T. Cartography: Thematic Map Design. 6 ed. Boston: McGraw Hill Higher Education, 2009.

DIAS, L.B.A. et al. Dengue: transmissão, aspectos clínicos, diagnóstico e tratamento. Simpósio: Condutas em enfermaria de clínica médica de hospital de média complexidade. Ribeirão Preto, v.1, cap. VI, 2010.

HORTA, M.A.P.; FERREIRA, A.P.; OLIVEIRA, R.B. et al. Os efeitos do crescimento urbano sobre o processo de saúde e repercussões da dengue: um estudo de caso. Revista Uniandrade, v. 14, n. 3, p. 201-216, 2013. https://doi.org/10.18024/1519-

5694/revuniandrade.v14n3p201-216

IBGE. INSTITUTO BRASILEIRO DE GEOGRAFIA E ESTATÍSTICA. Censo 2010 - População urbana e rural. Disponível em: $<\mathrm{ftp}$ //ftp.ibge.gov.br/Censos/Censo_Demogr afico_2010/indicadores_sociais_municipais/B rasil.zip> Tabela 1. Acesso em: 31 mai. 2016.

IBGE. INSTITUTO BRASILEIRO DE GEOGRAFIA E ESTATÍSTICA. Censo 2010 - Saneamento. Disponível em: $<\mathrm{ftp}$ //ftp.ibge.gov.br/Censos/Censo_Demogr afico_2010/indicadores_sociais_municipais/B rasil.zip> Tabela 7. Acesso em: 31 mai. 2016.

MEDRONHO, R. A. Dengue e o ambiente urbano. Revista Brasileira de Epidemiologia. São Paulo, v. 9, n. 2, p. 159161, jun. 2006.

MENDONÇA, F.A.; SOUZA, A.V. e; DUTRA, D.A. Saúde pública, urbanização e dengue no Brasil. Soc. nat. (Online), Uberlândia, v. 21, n. 3, p. 257-269, dez. 2009.

MISSIER, P. et al. Tracking Dengue Epidemics using Twitter Content Classification and Topic Modelling. Cornell University Library. 2016. https://doi.org/10.1007/978-3-319-46963$\underline{87}$ 
RESENDES, A.P.C. et al. Determinação de áreas prioritárias para ações de controle da dengue. Rev Saúde Pública, Rio de Janeiro, v.44, n.2, p.274-82, 2010.

SAZONOV, I.; KELBERT, M.; GRAVENOR, M.B. The speed of epidemic waves in a onedimensional lattice of sir models. Mathematical Modelling of Natural Phenomena. v.3, n. 04, p. 28-47, 2008. https://doi.org/10.1051/mmnp:2008069

SILVA, M. et al. A dengue no brasil e as políticas de combate ao Aedes aegypti: da tentativa de erradicação ás políticas de controle. Revista Brasileira de Geografia Médica e da Saúde, Jataí, v. 3, n.6, p.163175, jun. 2008.

SIQUEIRA, J.B. et al. Spatial point analysis based on dengue surveys at household level in central Brazil. BMC Public Health, v.8, p.361, 2008. https://doi.org/10.1186/1471$\underline{2458-8-361}$

SUAREZ, R. et al . An ecosystem perspective in the socio-cultural evaluation of dengue in two Colombian towns. Cad. Saúde Pública, Rio de Janeiro, v. 25 , supl. 1, p. S104-S114, jan. 2009.

TAUIL, P.L. Urbanização e ecologia do dengue. Cad. Saúde Publica. Brasília, v. 17, p. 99-102, 2001.

WHO. World Health Organization. Annual Cases Reported of Dengue, Washington, 2016. 